\title{
Forecasting of the composite dose for organs at risk and solid targets with random movements during different image-guided scenarios of the photon radiation therapy. Solution for the Varian therapeutic line
}

\author{
Adam Ryczkowski ${ }^{1,2}$, Tomasz Piotrowski ${ }^{1,2}$ \\ ${ }^{1}$ Department of Medical Physics, Greater Poland Cancer Centre, Poznań, Poland \\ ${ }^{2}$ Department of Electroradiology, Poznań University of Medical Sciences, Poznań, Poland
}

\begin{abstract}
Background: This study aims to develop a useful tool for robust plan analysis which includes the effects of soft tissue deformations on simulated dose distributions. The solution was benchmarked in the light of the commercial method implemented in Eclipse $^{\mathrm{TM}}$ treatment planning system (TPS).

Materials and methods: Study was carried out on data of one patient with prostate-restricted cancer. The workflow of the procedure developed focused on three executive elements: in-house script to create a set of artificial CT images and for movement simulation of the CTV; the Velocity ${ }^{\mathrm{TM}}$ software for the calculations of the deformation matrixes and, then, to generate deformed CT sets; the Eclipse ${ }^{\mathrm{TM}}$ TPS for dose re-calculations and analysis. Two scenarios were examined — first when the recalculation was done for the original geometry and second, when the isocentre from the original plan geometry was moved according to the movement of the CTV. The dose distributions were analysed on dose volume histograms (DVHs) in the light of the results obtained from the method implemented in the Eclipse ${ }^{\mathrm{TM}}$ TPS.

Results: The DVHs from our methods are more informative than the DVH from commercially implemented tools. For the first scenario, the highest impact on dose uncertainty has boundary positions of the CTV to the CTV-PTV margin. Using the second scenario, it is the relation of the CTV position to the whole body that has the highest effect on dose uncertainty.

Conclusion: Our method enables a more accurate analysis of the treatment plan robustness than the method currently implemented in Eclipse ${ }^{\mathrm{TM}}$ TPS.

Key words: robust plan analysis; robustness; robust evaluation; prostate radiotherapy

Rep Pract Oncol Radiother 2021;26(3):489-494
\end{abstract}

\section{Introduction}

The most used radiation therapy treatments based on treatment plans, in which the delineation of the target implicates the necessary definition of the margin around the clinical target volume (CTV). CTV with this specific, geometrical, buffer zone is defined as planning target volume (PTV). The size of the CTV-PTV margin depends on the internal movement of the tumour and the errors caused by the patient set-up and the dose delivery methods or specific dose distributions obtained during treatment. Correctly applied CTV-PTV margin should provide the delivery of the planned

Address for correspondence: Adam Ryczkowski, MSc, Department of Medical Physics, Greater Poland Cancer Centre, Garbary 15, 61-866 Poznan, Poland; e-mail: adam.ryczkowski@wco.pl 
dose to the CTV during the treatment [1-4]. Taking into account the accuracy of dose delivery - the size of the CTV-PTV margin depends on the radiation therapy method selected in the treatment and on the image-guidance scenarios used during the treatment. Numerous investigators show that increasing the frequency of image guidance (IG) allows for smaller CTV-PTV margins [5-8], or that the magnitude of margins depends on the modality used for IG $[9,10]$.

The safety of the PTV-based treatments makes two main assumptions. First is that moderate changes in patient position or anatomy do not affect the dose distribution and the second that the effects of the interplay between intra-fractional motion and dynamic fluence patterns are negligible [11]. Taking into account $5 \%$ as the acceptable dosimetric accuracy between planned and delivered doses [12] a lot of studies confirmed that the safety PTV-based concept is suitable for photon radiation therapy [13-18]. Nevertheless, assuming the correctness of the dose delivery at the accelerator level, the distribution of the dose uncertainties could differ for different IG scenarios used during therapy. Therefore, the analysis of the plan robustness on the potential movement of the CTV and the deformations of the tissues caused from this movement is one of the crucial methods to obtain information about possible dose distributions during correctly performed radiation therapy.

There are two methods of robust plan analysis in the Eclipse ${ }^{\mathrm{TM}}$ treatment planning system (TPS) (Varian Medical Systems, Palo Alto, CA, USA) that are available in the "Plan Uncertainty Parameters" dialogue box (PUP). First of them estimates how a change in patient set-up may affect the dose distribution, the other one forecasts the effect on the dose distribution if the target moves to other structures. While the first method simulates the dose distributions during the radiation therapy managed and controlled by classical IG, such as no action level (NAL) protocol, the other one tries to simulate the dose distributions obtained during daily IG procedures. Unfortunately, these scenarios do not include in the final budget of the dose distributions the effect caused by deformations of the soft tissues that are accompanying the change of position of the CTV.

This study aimed to present the solution, based mainly on Varian commercial products, for robust plan analysis, which includes the effects of the soft tissue deformations on the simulated dose distributions.

\section{Materials and methods}

Study was carried out on data of one patient with prostate-restricted cancer. The workflow of the developed procedure focused on three executive elements:

- in-house script for movement simulation of the CTV written in the Python programming language;

- the Velocity ${ }^{\mathrm{TM}}$ software (Varian Medical Systems, Palo Alto, CA, USA) for calculations of the deformation matrixes;

- the Eclipse ${ }^{\mathrm{TM}}$ TPS for dose re-calculations and analysis.

Figure 1 shows the general workflow of the authors' solution.

The contours, previously prepared by a radiation oncologist on original set of CT images, are used by an in-house script to create a set of artificial set of CT images - $\mathrm{aCT}_{(0,0,0)}$. Our script used external (body), bones and CTV contours to create masks on CT images (Fig. 1. - In-house engine section). The pixel value assigned to the body was $0 \mathrm{HU}$, the pixel value assigned to the bone and CTV was 1000 HU. In the next step, the in-house script simulates on $\mathrm{aCT}_{(0,0,0)}$ all possible positions of the CTV and, finally, generates the group of aCT sets that differ in the position of the CTV in each direction ( $x, y$ and $\mathrm{z})-\mathrm{aCT}_{(\mathrm{x}, \mathrm{z}, \mathrm{l})}$. The positions of the CTV are quantified by the pixel value (i.e. $0.98 \mathrm{~mm}$ ) and are ranged in the CTV-PTV margin (10 $\mathrm{mm}$ buffer zone added spherically to CTV).

All of the aCT sets are uploaded to the Velocity software where they are used to generate the deformation matrixes and then to generate deformed CT sets (dCT) based on the original (planning) CT. The whole procedure was based on manual scripting in the Velocity ${ }^{\mathrm{TM}}$ software and included respectively:

- rigid registrations between $\mathrm{aCT}_{(0,0,0)}$ and any other aCT $\mathrm{T}_{(x, y, z)}$;

- deformable registrations between $\mathrm{aCT}_{(0,0,0)}$ and any other $\mathrm{aCT}_{(\mathrm{x}, \mathrm{y}, \mathrm{z})}$ to generate deformation matrixes $\left(\mathrm{DM}_{(\mathrm{x}, \mathrm{y}, \mathrm{z})}\right)$ caused from different positions of CTV on $\mathrm{aCT}_{(0,0,0)}$ and $\mathrm{aCT}_{(\mathrm{x}, \mathrm{y}, \mathrm{z})}$;

- appling $\mathrm{DM}_{(x, y, z)}$ to original set of CT images and generating deformed $\mathrm{CT}$ sets $(\mathrm{dCT})$. The 


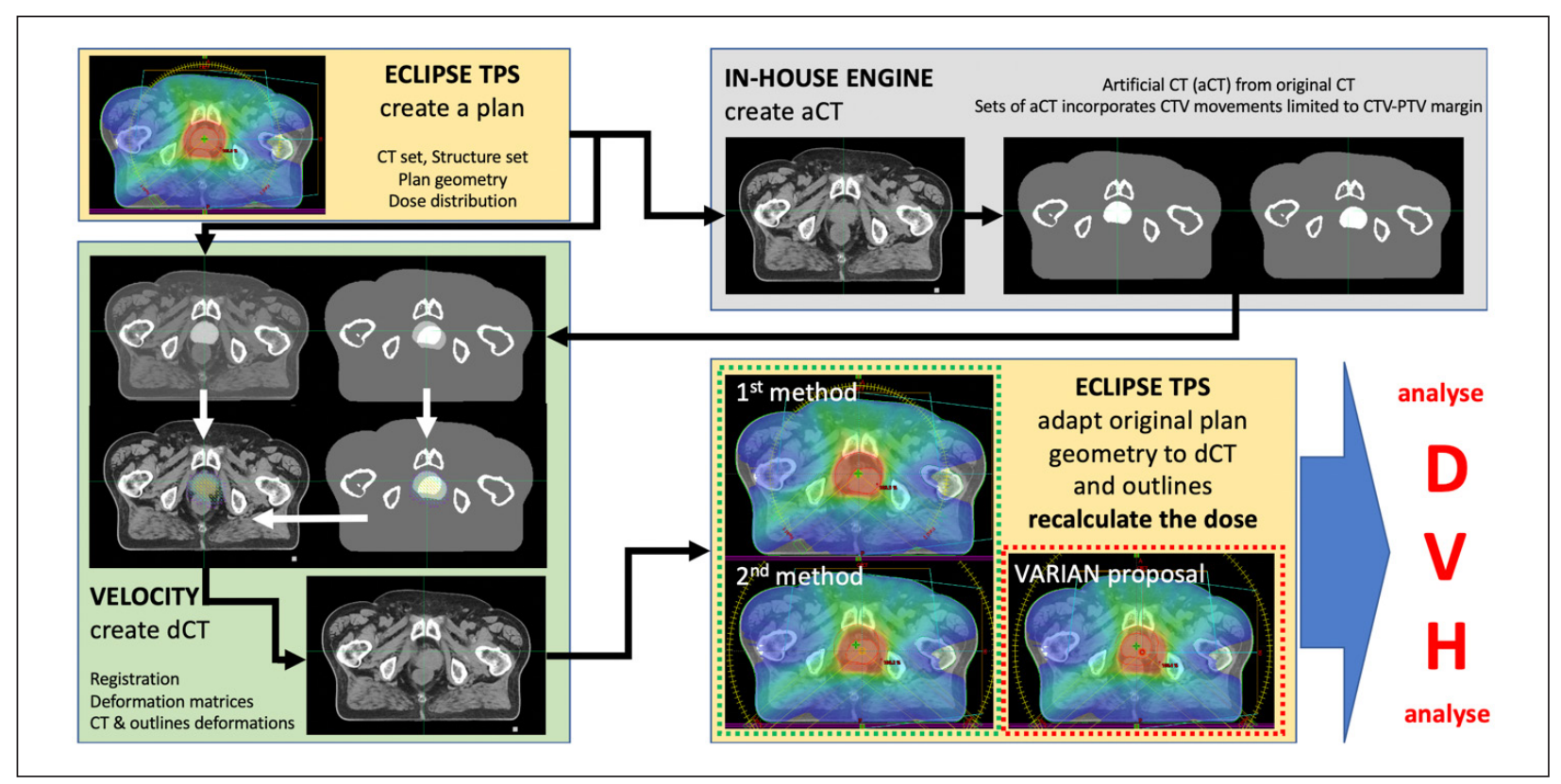

Figure 1. General workflow of the authors' solution. aCT — artificial set of CT images; dCT — deformed set of CT images; $1^{\text {st }}$ method - re-calculation is done for the original geometry (simulation of IG based on the bony anatomy); $2^{\text {nd }}$ method - the isocentre from original plan geometry is moved according to the movement of the CTV (simulation of IG based on soft tissues)

same deformation matrixes were used to deform OARs contours. The deformable multipass algorithm was used for the last two steps of the procedure [19].

The dCT sets are moved to the Eclipse software where they are linked with the original plan geometry. For each dCT set, the doses were re-calculated. Two possible scenarios of IG procedures were considered $[7,20]$ when doses were re-calculated:

- the first method: re-calculation is done for the original geometry. It simulates IG based on the bony anatomy (not included bone-independent movements of the CTV) - Figure 2A;

- the second method: the isocentre from the original plan geometry is moved according to the movement of the CTV (simulates IG based on soft tissues) - Figure 2B.

Finally, the dose distributions for the CTV, rectum and bladder for both scenarios were analysed used dose-volume histogram (DVH) metrics, in the same way as is possible for the currently developed tool in Eclipse ${ }^{\mathrm{TM}}$ TPS (Fig. 2C).

\section{Results}

There is about 1700 possible deformations of the original set of CT images (dCT). This value was calculated by a script which moves a CTV mask in the step of one voxel in all directions and checks if it is still inside PTV. Number of possible positions of the CTV mask is dependent on the CTV-PTV margin. Twenty-six of them were selected as the most significant for the results. These were the most extreme positions of the CTV inside the PTV in each direction $(x, y, z)$ and their combinations $(x y, y z$, $\mathrm{xz}, \mathrm{xyz}$ ). The dCT sets, obtained in this way, were exported to Eclipse ${ }^{\mathrm{TM}}$ TPS where the doses were re-calculated for them according to two proposed scenarios. Additionally, the original treatment plan has been simulated by the Varian's formula implemented in the System. Figure 3 shows the uncertainty of the dose distribution in CTV, bladder and rectum for two scenarios of IG, calculated by authors' solution and by the tool commercially implemented in Eclipse ${ }^{\mathrm{TM}}$ TPS.

\section{Discussion}

The term "robustness" in the light of the radiotherapeutic plans is considered in two ways. First of them is a robust plan optimisation - the set of procedures that allows minimising potential uncertainties of the dose distribution during the plan preparation. During the robust optimisation, a lot 
$1^{\text {st }}$ uncertainty simulation method

while IG based on bone anatomy is used during RT
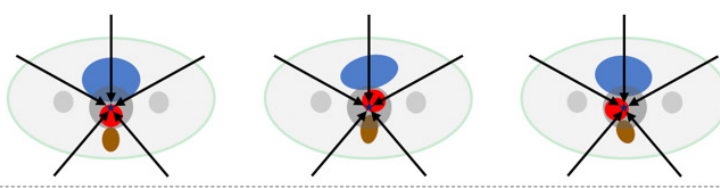

B

$2^{\text {nd }}$ uncertainty simulation method

while IG based on soft tissues (CTV) is used during RT
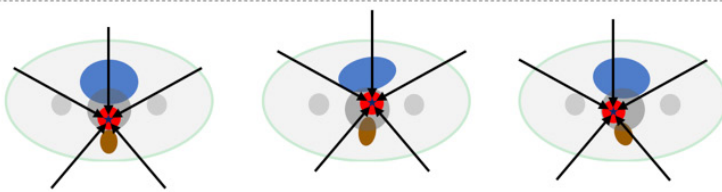

Varian proposal for uncertainty simulation good for uncertainty simulation when IG based on No Action Level protocols is used during RT
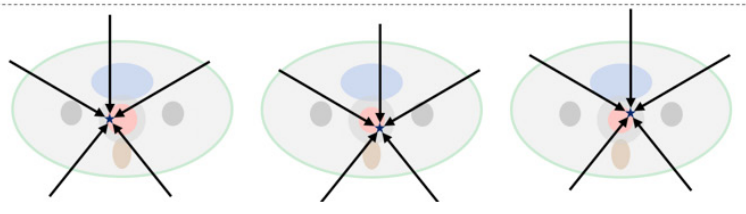

Legend

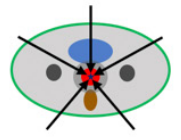

- red circle - Clinical Target Volume

- grey circle - Planning Target Volume

grey eclipse and green outline - patient body

- black circles - femoral heads

blue eclipse - bladder

- brown eclipse - rectum

$\rightarrow$ arrow - beam direction

$\star \quad$ star -isocentre

Figure 2. Schemes of uncertainty simulations used in the study
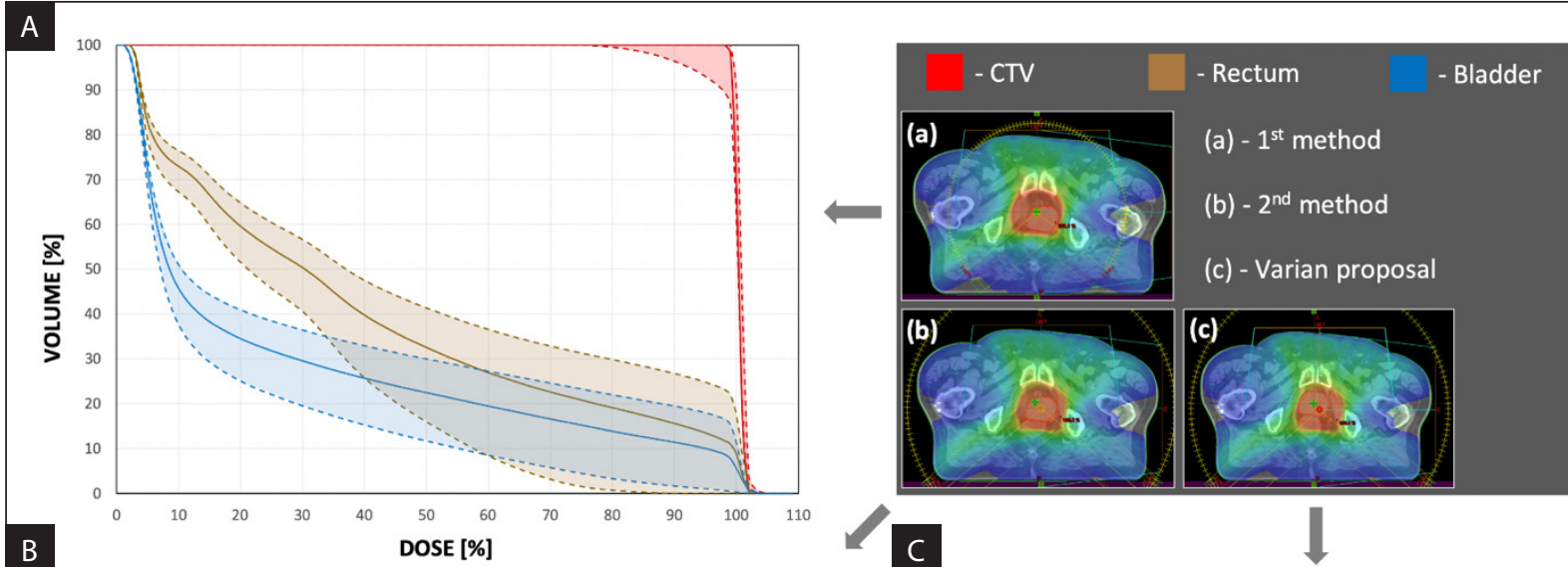

B
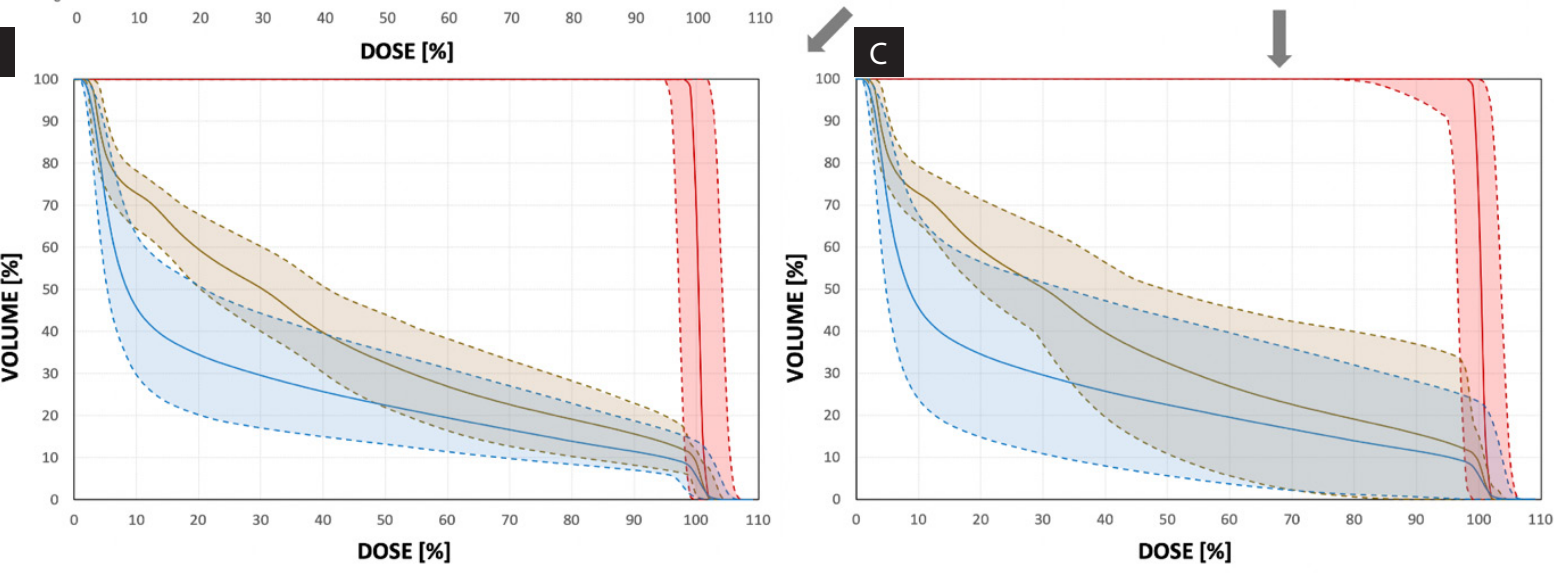

Figure 3. The uncertainty of the dose distribution in CTV, bladder and rectum for two scenarios of IG, calculated by authors' solution (A, B) and the Varian's formula implemented in the Eclipse ${ }^{\mathrm{TM}}$ TPS (C) 
of scenarios were considered. Each scenario represents a possible treatment course and should include a specification of all the errors that are needed to calculate the final dose distribution [21]. The second way is a robust plan analysis - the analysis of the potential uncertainties of dose distribution for a single plan prepared in a conventional way (i.e., patient in a fixed nominal position and considering CTV-PTV margins). In other words, the intention of a robust optimisation is a reduction of potential uncertainties of doses on the level of plan preparation and, then, proper irradiation. In contrast, a robust plan analysis answers the question of how accurate is the treatment based on the plan created conventionally. This study focuses on a robust plan analysis.

Eclipse $^{\mathrm{TM}}$ TPS has a tool for calculation of the dose uncertainty for conventionally prepared treatment plans. The procedure of uncertainty simulation in this tool is quite simplified and based on moving the whole patient's body around the isocentre of the treatment (Fig. 2C). The scenarios of available simulations there do not include the changes of dose distribution caused by deformations of the soft tissues that are accompanying the shift in the position of the CTV. Moreover, they do not precisely simulate the different scenarios of IG that have an impact on the difference in doses. When daily is IG based on the bony anatomy, the isocentre of the treatment is fixed in the same geometrical place for each fraction. Potential differences in the fraction doses in the tumour (CTV) as well as in organs at risk (OARs) are caused by the movement of the CTV and different filling of OARs (i.e. bladder, rectum) during fractions (Fig. 2A). In the case of daily IG based on soft tissues, the isocentre of the treatment depends on the current position of the CTV before the fraction dose delivery. While the impact of OARs deformation on the doses is similar, the inter-fraction tracking of CTV position reduces effectively the dose differences in the CTV caused by different positions of the CTV. Nevertheless, the different distance between the skin and the isocentre may cause a difference in doses (Fig. 2B).

The observations listed above motivated us to develop an in-house solution that enabled more precise simulations of dose uncertainties. In our solution, as in the Varian's tool, the dose uncertainty analysis is based on the information gathered on the DVH. The DVHs from our method are more informative than the DVH from commercially implemented tools. For the first scenario (IG based on bony anatomy, Fig. 3A), the highest impact on dose uncertainty has boundary positions of the CTV to the CTV-PTV margin and relocation of the CTV that causes changes of OARs volumes that receive high doses. For the second scenario (IG based on soft tissues, Fig. 3B), dose uncertainty is affected to the highest degree by the CTV position in relation to the whole body and relocation of the isocentre that causes changes in the general dose distribution (visible for OARs volume receiving low and intermediate doses). The current version of our procedure is limited to the solid (nondeformed) CTVs.

Except the in-house engine used to generate aCT sets with different position of the CTV, the tools (i.e. Velocity ${ }^{\mathrm{TM}}$ and Eclipse ${ }^{\mathrm{TM}}$ ) used in our simulations are developed by the Varian Medical Systems. They are being used commercially in a lot of centres based on the Varian's radiotherapeutic line. Moreover, it is possible to automatise the procedures carried out in Velocity ${ }^{\mathrm{TM}}$ and Eclipse ${ }^{\mathrm{TM}}$ by scripting language implemented in these programs. Due to this fact, it is easy to implement this solution in centres based on Varian's line.

\section{Conclusion}

The proposed method of simulating the uncertainty of dose distributions enables a more accurate analysis of the treatment plan robustness than the method currently implemented in Eclipse ${ }^{\mathrm{TM}}$ TPS.

\section{Conflict of interest}

The authors declare that they have no conflict of interest.

\section{Funding}

The authors declare that they have no financial connection.

\section{References}

1. Prescribing, Recording, and Reporting Proton-Beam Therapy. J. ICRU. 2019; 7(2): 1-8, doi: 10.1093/jicru_ndm021.

2. The Royal College of Radiologists, Society and College of Radiographers, Institute of Physics and Engineering in Medicine. On target: ensuring geometric accuracy in radiotherapy. The Royal College of Radiologists, London 2008.

3. van Herk M, Remeijer P, Rasch C, et al. The probability of correct target dosage: dose-population histograms for 
deriving treatment margins in radiotherapy. Int J Radiat Oncol Biol Phys. 2000; 47(4): 1121-1135, doi: 10.1016/ s0360-3016(00)00518-6, indexed in Pubmed: 10863086.

4. Korevaar EW, Habraken SJM, Scandurra D, et al. Practical robustness evaluation in radiotherapy - A photon and proton-proof alternative to PTV-based plan evaluation. Radiother Oncol. 2019; 141: 267-274, doi: 10.1016/j.radonc.2019.08.005, indexed in Pubmed: 31492443.

5. Gupta M, Gamre P, Kannan S, et al. Effect of imaging frequency on PTV margins and geographical miss during image guided radiation therapy for prostate cancer. Pract Radiat Oncol. 2018; 8(2): e41-e47, doi: 10.1016/j. prro.2017.09.010, indexed in Pubmed: 29122556.

6. Zeidan OA, Langen KM, Meeks SL, et al. Evaluation of image-guidance protocols in the treatment of head and neck cancers. Int J Radiat Oncol Biol Phys. 2007; 67(3): 670-677, doi: 10.1016/j.ijrobp.2006.09.040, indexed in Pubmed: 17197123.

7. Piotrowski T, Kaczmarek K, Bajon T, et al. Evaluation of image-guidance strategies for prostate cancer. Technol Cancer Res Treat. 2014; 13(6): 583-591, doi: 10.7785/ tcrtexpress.2013.600258, indexed in Pubmed: 24000985.

8. Garibaldi C, Fodor C, Riva G, et al. Cone-beam CT-based inter-fraction localization errors for tumors in the pelvic region. Phys Med. 2018; 46: 59-66, doi: 10.1016/j. ejmp.2018.01.011, indexed in Pubmed: 29519410.

9. Yartsev S, Bauman G. Target margins in radiotherapy of prostate cancer. Br J Radiol. 2016; 89(1067): 20160312, doi: 10.1259/bjr.20160312, indexed in Pubmed: 27377353.

10. McPartlin AJ, Li XA, Kershaw LE, et al. MR-Linac consortium. MRI-guided prostate adaptive radiotherapy - A systematic review. Radiother Oncol. 2016; 119(3): 371-380, doi: 10.1016/j.radonc.2016.04.014, indexed in Pubmed: 27162159.

11. Yock AD, Mohan R, Flampouri S, et al. Robustness Analysis for External Beam Radiation Therapy Treatment Plans: Describing Uncertainty Scenarios and Reporting Their Dosimetric Consequences. Pract Radiat Oncol. 2019; 9(4): 200-207, doi: 10.1016/j.prro.2018.12.002, indexed in Pubmed: 30562614.

12. Determination of absorbed dose in a patient irradiated by beams of $X$ or gamma rays in radiotherapy procedures. ICRU Report 24. International Commission on Radiation Units and Measurements, Bethesda 1976.
13. Engelsman M, Damen EM, De Jaeger K, et al. The effect of breathing and set-up errors on the cumulative dose to a lung tumor. Radiother Oncol. 2001; 60(1): 95-105, doi: 10.1016/s0167-8140(01)00349-8, indexed in Pubmed: 11410310.

14. Yu CX, Jaffray DA, Wong JW. The effects of intrafraction organ motion on the delivery of dynamic intensity modulation. Phys Med Biol. 1998; 43(1): 91-104, doi: 10.1088/0031-9155/43/1/006, indexed in Pubmed: 9483625.

15. Bortfeld T, Jiang SB, Rietzel E. Effects of motion on the total dose distribution. Semin Radiat Oncol. 2004; 14(1): 41-51, doi: 10.1053/j.semradonc.2003.10.011, indexed in Pubmed: 14752732.

16. Bortfeld T, Jokivarsi K, Goitein M, et al. Effects of intra-fraction motion on IMRT dose delivery: statistical analysis and simulation. Phys Med Biol. 2002; 47(13): 2203-2220, doi: 10.1088/0031-9155/47/13/302, indexed in Pubmed: 12164582.

17. Adamczyk M, Kruszyna-Mochalska M, Rucińska A, et al. Software simulation of tumour motion dose effects during flattened and unflattened ITV-based VMAT lung SBRT. Rep Pract Oncol Radiother. 2020; 25(4):684-691, doi: 10.1016/j. rpor.2020.06.003, indexed in Pubmed: 32581656.

18. Court LE, Seco J, Lu XQ, et al. Use of a realistic breathing lung phantom to evaluate dose delivery errors. Med Phys. 2010;37(11): 5850-5857, doi: 10.1118/1.3496356, indexed in Pubmed: 21158297.

19. Pukala J, Johnson PB, Shah AP, et al. Benchmarking of five commercial deformable image registration algorithms for head and neck patients. J Appl Clin Med Phys. 2016; 17(3): 25-40, doi: 10.1120/jacmp.v17i3.5735, indexed in Pubmed: 27167256.

20. Jodda A, Piotrowski T, Urbański B, et al. Relations between dose cumulated in organs at risk and treatment based on different image-guidance strategies of cervical cancer. Phys Med. 2019; 57: 183-190, doi: 10.1016/j. ejmp.2019.01.008, indexed in Pubmed: 30738524.

21. Hernandez V, Hansen CR, Widesott $L$, et al. What is plan quality in radiotherapy? The importance of evaluating dose metrics, complexity, and robustness of treatment plans. Radiother Oncol. 2020; 153: 26-33, doi: 10.1016/j.radonc.2020.09.038, indexed in Pubmed: 32987045 . 